\title{
Nonuniversal dynamics of dimer growing interfaces
}

\author{
M. D. Grynberg \\ Departamento de Física, Universidad Nacional de La Plata, (1900) La Plata, Argentina
}

(Received 27 June 2007; published 26 September 2007)

\begin{abstract}
A finite temperature version of body-centered solid-on-solid growth models involving attachment and detachment of dimers is discussed in $1+1$ dimensions. The dynamic exponent of the growing interface is studied numerically via the spectrum gap of the underlying evolution operator. The finite size scaling of the latter is found to be affected by a standard surface tension term on which the growth rates depend. This nonuniversal aspect is also corroborated by the growth behavior observed in large scale simulations. By contrast, the roughening exponent remains robust over wide temperature ranges.
\end{abstract}

DOI: 10.1103/PhysRevE.76.031605 PACS number(s): 81.15.Aa, 05.10.Gg, 02.50.-r, 75.10.Jm

\section{INTRODUCTION}

In studying statistical aspects of nonequilibrium surfaces the onset of scaling regimes at both large time and length scales has enabled one to characterize a vast body of growth processes in terms of universality classes [1]. In analogy to equilibrium phase transitions, there is consensus in that the late evolution stages of these processes are controlled by a set of scaling exponents stemming ultimately from the symmetries and conservations laws of the underlying growth rules. A basic quantity of interest investigated extensively in this context concerns the roughness or surface width $W(L, t)$ developed by growth fluctuations at a given time $t$ when starting from an initially flat substrate of typical length $L$. Based on a wide range of theoretical and numerical studies it can be argued that $W$ scales as $[1,2]$

$$
W(L, t)=L^{\zeta} f\left(t / L^{z}\right),
$$

with a universal scaling function behaving as $f(x) \sim x^{\zeta / z}$ for $x \ll 1$, whereas for $x \gg 1$ it remains constant. Consequently, for $t \gg L^{z}$ the width saturates as $L^{\zeta}$ while growing as $t^{\zeta / z}$ in the thermodynamic limit. The roughening exponent $\zeta$ measures the stationary dependence of the surface width on the typical substrate size while $z$, frequently referred to as the dynamic exponent, gives the fundamental scaling between length and time.

In this work we focus on rather unusual scaling properties studied in recent years both in one [3-5] and two dimensional interfaces [6] by means of discrete models of surface growth. For simplicity, here we consider a body-centered solid-on-solid (BCSOS) version of these [5], limiting height differences between neighbors to \pm 1 rather than to $0, \pm 1$, as in restricted SOS realizations [3,4]. Our basic kinetic steps (depicted schematically in Fig. 1), involve adsorption and desorption, possibly after recombination, of dimers only. Attempts of desorption can take place whether or not the selected pair of adjacent monomers arrived together. Therefore, the rule for evaporation allows for reconstitution of dimers, a crucial feature, so their identity is not maintained during the growth process.

It is important to note that throughout the stochastic evolution the parity of the number of monomers (eventually isolated) is conserved at each height level of the surface. The implications of this "evenness" nonlocal constraint on the scaling exponents are far reaching $[3,6]$, and in the one dimensional (1D) case have been analyzed in terms of even visiting random walks. In this latter representation, interface configurations are thought of as Brownian paths whose locations (i.e., height levels) are visited an even number of times before the walk terminates on a given time interval (here playing the role of the substrate length). In marked contrast to normal random walks, the evenness constraint introduces highly correlated movements giving rise to an anomalous (subdiffusive) mean square displacement, which in the interface language means a saturated width scaling not as $L^{1 / 2}$ but rather as $L^{1 / 3}[3]$. Also, the dynamic exponents $z$ obtained numerically for these [3-5] and related globally constrained systems $[9,10]$ are definitely different from those of usual monomer type interfaces, irrespective of the later evolving towards equilibrium or nonequilibrium stationary regimes (as exemplified, respectively, by the Edward-Wilkinson [7] (EW) and Kardar-Parisi-Zhang [8] (KPZ) universality classes). These anomalous aspects of nonlocal constraints (a)

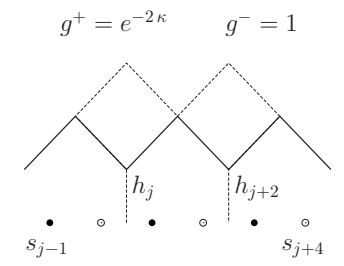

(c)

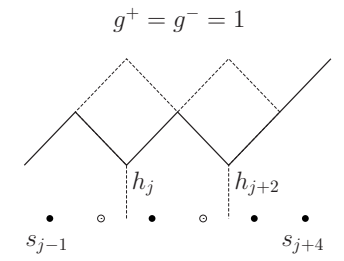

(b)

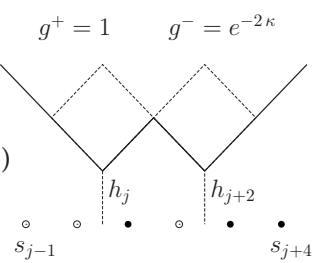

(d)

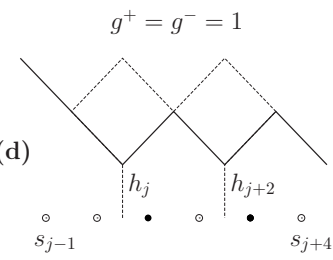

FIG. 1. The four relevant deposition-evaporation cases and their respective rates $g^{+}, g^{-}$for a one dimensional BCSOS dimer growing interface. Adding or removing a dimer (denoted by dotted lines) at columns $h_{j}, h_{j+2}$ can be viewed as flipping the spin- $\frac{1}{2}$ quartet $s_{j}, s_{j+1}, s_{j+2}, s_{j+3}$. The involved rates depend on the change of surface tension (2), which is in turn determined by the spin (or slope) states of $s_{j-1}$ and $s_{j+4}$ [see Eqs. (3) and (6)]. Each situation is depicted schematically from (a) to (d). 
should not be regarded as purely academic. In fact, in catalytic surface processes the interplay between the substrate geometry and the shape of the intervening objects does matter. In particular, dimers become relevant in the roughening dynamic of vicinal surfaces, which only allows deposition and evaporation of diatomic molecules [6].

To our knowledge, there are no available phenomenological equations of growth (e.g., KPZ, EW), suitable to describe interface fluctuations arising from deposition-evaporation of composite particles. The manner in which nonlocalities could be incorporated in that continuum limit is by far not clear. However, in probing the robustness of the dissociative dimer models referred to above some progress can be made by introducing a continuously tunable parameter without affecting either the symmetries or the conservation laws of their dynamics. Specifically, we consider a finite temperature extension of 1D dimer growing interfaces, which for the ease of our numerical analysis (Sec. III A), is here taken under detailed balance conditions. Following Ref. [11], this is readily done through a standard surface tension term associated to configurational energy scales which discourage the development of strong fluctuations, besides those already prevented by both BCSOS and evenness constraints. In spite of these severe restrictions, they should not impede us in evaluating scaling exponents, as equilibrium surfaces at finite temperatures are always rough in $1 \mathrm{D}$. In the case of 1D-monomer growing interfaces, the surface tension is not relevant to drive them out of their universality classes, though interestingly in 2D it can change the nonlinear term sign of the KPZ equation thus inducing rough-to-rough transitions $[11,12]$. Surprisingly, for dimers it will turn out that the combined effects of global constraints and surface tension entail significant changes in the $z$ dynamic exponent, suggesting rather a nonuniversal temperature dependent value. In fact, nonuniversal aspects were already observed over wide temperature ranges in the monomer systems studied in Refs. [11,12], although they were ascribed to finite size effects which become particularly severe in nearing the equilibrium roughening transition temperature $T_{R}$ [13]. However, as mentioned above, in our $1 \mathrm{D}$ case $T_{R}$ is strictly zero and in practice all equilibrium correlation lengths can be fairly bounded as long as temperatures are not taken too low. In this sense, it is worth mentioning that steep variations of $z$ will already appear within high temperature regimes (Sec. III). By contrast, the roughening exponent $\zeta$ remains robust as differences with respect to its vanishing tension limit $(\zeta$ $\sim 1 / 3$ ), will basically merge with our numerical errors. Since by definition $\zeta$ is a stationary index, it is just the nonequilibrium dynamics that is being strongly affected, as we shall see.

Turning to methodological issues, as is known, the dynamic scaling hypothesis referred to in Eq. (1) is usually put forward to determine both $\zeta$ and $z$ in a joint way by simulating the growth dynamics over different substrate sizes. With the aim of obtaining an independent (separate) evaluation of these exponents, in addition to this standard procedure we will also exploit the known equivalence between BCSOS models and interacting gases of hard-core particles [14]. Following the thread of ideas given in [15], we will recast the Metropolis operator that rules our growth simulations in terms of a quantum spin representation. This latter lends itself more readily for a finite size scaling analysis of the gap of the Metropolis operator which ultimately is related to the $z$ dynamic exponent. On the one hand this technique avoids the problem of dealing with long transient regimes though on the other hand is limited severely by the affordable substrate sizes. For now let us simply remark that already modest lengths are able to yield clear finite size trends over wide temperature ranges. To complement our approach, at low temperature regimes (where correlation lengths exceed the sizes reachable by exact diagonalization), we will rely on numerical simulations of much larger systems which along with Eq. (1) will further support the nonuniversal picture.

The layout of this work is organized as follows. In Sec. II we construct the quantum spin analogy of the standard Metropolis dynamic and briefly touch upon symmetries and conservation laws. By means of an ulterior nonunitary spin rotation, this results in a symmetric representation of the Metropolis operator. This simplifies considerably the subsequent numerical analysis of Sec. III in which the spectrum gap of this operator is obtained via standard recursive techniques [16]. The evaluation of dynamic exponents is then extended to low temperature regimes using standard Monte Carlo simulations. Finally, Sec. IV contains a summarizing discussion along with some remarks on extensions of this work.

\section{DYNAMIC AND REPRESENTATIONS}

As usual, the state of a solid-on-solid interface is represented by a set of single-valued functions $\left\{h_{j}(t)\right\}$ denoting height levels at positions $j=1, \ldots, L$ measured at a given time $t$ from a reference substrate of length $L$. As mentioned earlier, to prevent arbitrary bulk fluctuations we impose BCSOS constraints on these heights which hereafter are taken to satisfy $h_{j+1}-h_{j}= \pm 1, \forall j, t$, along with periodic boundary conditions (PBC). Growth or evaporation of the interface involves two particles (dimers) at the top of columns $h_{j}, h_{j+2}$ which, to comply with the above restrictions, ought both to be local extrema of the evolving interface. More specifically, deposition (evaporation) events $h_{j}, h_{j+2} \rightarrow h_{j}$ $+2, h_{j+2}+2\left(h_{j}, h_{j+2} \rightarrow h_{j}-2, h_{j+2}-2\right)$ can only occur at two consecutive local minima (maxima) of the heights set (see Fig. 1). We stress that evaporation takes place regardless if these maxima were created together or not, so dimers can dissociate. Also note that the number of heights at a given level preserves its parity throughout.

We want the transition rates of these processes to depend on the surface tension $\sigma$ referred to in Sec. I. In turn, any model that associates energies to height differences should provide a plausible description of $\sigma$. Due to our BCSOS choice $(\Delta h= \pm 1)$, evidently the simplest form of $\sigma$ should assign an energy $\epsilon>0$ to each double facet exposed between columns $h_{j}$ and $h_{j+2}$. Since $h_{j+2}-h_{j}=0, \pm 2$, this may be studied by defining

$$
\sigma=\frac{\epsilon}{2} \sum_{j}\left|h_{j+2}-h_{j}\right|,
$$

which simply counts the total number of double facets in a given interface configuration. Therefore, we can construct a 
standard Metropolis process at temperature $T$ after introducing the following transition probability rates

$$
g^{ \pm}\left(h_{j}, h_{j+2} \rightarrow h_{j} \pm 2, h_{j+2} \pm 2\right)=\min \left\{e^{-\Delta_{j} \sigma / T}, 1\right\},
$$

where $\Delta_{j} \sigma$ is the change in surface tension upon depositing $\left(g^{+}\right)$or removing $\left(g^{-}\right)$a dimer at $h_{j}, h_{j+2}$ (henceforth, the Boltzmann constant $k_{B}$ is set equal to one). The four possible scenarios determining the values of these rates, which by construction obey detailed balance (that is, $g^{ \pm}(\Delta \sigma / T) / g^{ \pm}($ $-\Delta \sigma / T)=e^{-\Delta \sigma / T}$ ), are schematized in Fig. 1. Clearly, in the high temperature limit we recover the dimer model considered in [5], whereas $T \rightarrow 0^{+}$serves to favor smooth states over long transient regimes.

It is often more practical to work in terms of slopes rather than with interface heights, so in what follows we will employ the known mapping between BCSOS and hard-core particle dynamics [14]. This correspondence is easily visualized in Fig. 1, which simply associates the height differences $h_{j}$ $-h_{j-1} \equiv s_{j}$ to particles $\left(s_{j}=1\right)$ or vacancies $\left(s_{j}=-1\right)$. Conversely, the interface heights are obtained as $h_{j}=\Sigma_{n \leqslant j} s_{n}$, modulo a constant level. In particular, in this picture the surface tension reduces to the Ising Hamiltonian

$$
\sigma=\frac{\epsilon}{2} \sum_{j} s_{j} s_{j+1},
$$

up to an irrelevant constant, whereas after some straightforward manipulations, the square deviation of the instantaneous average height $\bar{h}$ of a particular slope configuration $|s\rangle$, i.e., a given realization of the interface "width" $W_{|s\rangle}^{2} \equiv \Sigma_{j}\left(h_{j}\right.$ $-\bar{h})^{2} / L$, has the form

$$
W_{|s\rangle}^{2}=\frac{L^{2}-1}{6 L}+\frac{2}{L^{2}} \sum_{i<j} i(L-j) s_{i} s_{j} .
$$

Creating (eliminating) a dimer now amounts to a backwards $\bigcirc \bigcirc \bullet \bigcirc \bigcirc$ (forwards $\bigcirc \bigcirc \rightarrow \bigcirc \bigcirc$ ) move of two particles at a time, whereas from Eq. (4) it can be readily checked that in this representation the corresponding rates (3) just involve the neighboring states of the flipped quartet $s_{j}, s_{j+1}, s_{j+2}, s_{j+3}$, namely,

$$
g_{j}^{ \pm}=\min \left\{e^{ \pm \kappa\left(s_{j+4^{-}} s_{j-1}\right)}, 1\right\},
$$

with $\kappa \equiv \epsilon / T$ being from now on our inverse temperature parameter. Note also that under PBC the interface "magnetization" $\sum_{j} s_{j}$ vanishes at all evolution stages.

In addition, some constants of motion can be immediately identified. Evidently, the dimer dynamic exchanges two particles between four consecutive sites while changing the occupation of the involved next nearest neighbor locations by the same amount. If we think of these sites as being part of a four-partite lattice $\Lambda=\Lambda_{1}+\cdots+\Lambda_{4}(L / 2$ even), hence upon defining $S_{\alpha} \equiv \Sigma_{j \in \Lambda_{\alpha}} S_{j}$ as the magnetization of sublattice $\Lambda_{\alpha}$ it is clear that the set of dependent quantities $\left\{(-1)^{\alpha} S_{\alpha}\right.$ $\left.-(-1)^{\alpha^{\prime}} S_{\alpha^{\prime}}, \alpha, \alpha^{\prime}=1, \ldots, 4\right\}$ (of which only three are independent), is left invariant throughout. From a more fundamental point of view these conservations arise ultimately from continuous symmetries borne by the Metropolis operator controlling the probabilities of our slope states, and towards which we now turn.

\section{A. Metropolis operator}

As is known, the evolution operator of a Markovian process of the kind discussed so far can be constructed generically as [17]

$$
\begin{gathered}
\left\langle s^{\prime}|M| s\right\rangle=-R\left(s \rightarrow s^{\prime}\right), \quad s \neq s^{\prime}, \\
\langle s|M| s\rangle=\sum_{s^{\prime} \neq s} R\left(s \rightarrow s^{\prime}\right),
\end{gathered}
$$

where $R\left(s \rightarrow s^{\prime}\right)$ denotes the transition rate at which configuration $|s\rangle$ evolves to $\left|s^{\prime}\right\rangle$ per unit time. At least formally, this enables one to derive all subsequent probability distributions $|P(t)\rangle \equiv \sum_{s} P(s, t)|s\rangle$ from the action of the evolution operator on a given initial state, that is, $|P(t)\rangle=e^{-M t}|P(0)\rangle$ [17]. In our problem, $R\left(s \rightarrow s^{\prime}\right)=1, e^{-2 \kappa}$ for all compatibles $|s\rangle,\left|s^{\prime}\right\rangle$, and the specific form of $M$ can be readily found by interpreting the slope configurations $|s\rangle \equiv\left|s_{1}, \ldots, s_{L}\right\rangle$ as eigenstates of the $z$ component, say, of Pauli matrices $\vec{\sigma}_{1}, \ldots, \vec{\sigma}_{L}$ assigned to each slope site. For instance, using spin- $\frac{1}{2}$ raising and lowering operators $\sigma^{+}, \sigma^{-}$, the operational analog of Eq. (7) will then read

$$
\sum_{s, s^{\prime}, s \neq s^{\prime}} M_{s^{\prime}, s}\left|s^{\prime}\right\rangle\langle s|=-\sum_{j}\left(g_{j}^{+} A_{j}^{\dagger}+g_{j}^{-} A_{j}\right),
$$

where the adsorption (desorption) or double exchange operator $A_{j}^{\dagger}\left(A_{j}\right)$ acting on the $j$ quartet referred to above is simply

$$
A_{j}^{\dagger}=\sigma_{j}^{+} \sigma_{j+1}^{-} \sigma_{j+2}^{+} \sigma_{j+3}^{-} .
$$

Here, $g_{j}^{ \pm}$are thought of as diagonal operators in the $\sigma^{z}$ representation and whose elements are identified with the rates of Eq. (6). In this regard, the ordering of application in Eq. (9) is immaterial. As for the diagonal elements of Eq. (8), needed for conservation of probability, they basically count the number of ways in which a given configuration $|s\rangle$ can evolve to different states $\left|s^{\prime}\right\rangle$ by flipping an active quartet at a time. This can be properly tracked down in terms of number operators $\hat{n}=\sigma^{+} \sigma^{-}=\left(1+\sigma^{z}\right) / 2$ and weighting each probed quartet with its corresponding rate $\left(g^{ \pm}\right)$. The counterpart of Eq. (8) then becomes

$$
\begin{aligned}
\sum_{s} M_{s, s}|s\rangle\langle s|= & \sum_{j} g_{j}^{+}\left(1-\hat{n}_{j}\right) \hat{n}_{j+1}\left(1-\hat{n}_{j+2}\right) \hat{n}_{j+3} \\
& +\sum_{j} g_{j}^{-} \hat{n}_{j}\left(1-\hat{n}_{j+1}\right) \hat{n}_{j+2}\left(1-\hat{n}_{j+3}\right),
\end{aligned}
$$

which along with Eq. (9) completes the form of our Metropolis operator. Taking into account the spin algebra $\left[\sigma_{i}^{+}, \sigma_{j}^{-}\right]=\delta_{i, j} \sigma_{j}^{z},\left\{\sigma_{j}^{+}, \sigma_{j}^{-}\right\}_{+} \equiv 1$, the former finally reduces to

$$
M=\sum_{j}\left(g_{j}^{+} A_{j}^{\dagger}+g_{j}^{-} A_{j}\right)\left(A_{j}^{\dagger}+A_{j}-1\right) .
$$

By construction $M$ is a stochastic operator and therefore its ground state $\left|\Psi_{0}\right\rangle$ has vanishing eigenvalue and corresponds 
to the detailed balance solution of the problem, i.e., $\left|\Psi_{0}\right\rangle$ $\propto \sum_{s} e^{-(\kappa / 2) \sigma_{\{s\}}|S\rangle}$. Instead, its left ground state $\langle\tilde{\psi}|$ is an equally weighted linear combination of all reachable $\langle s|$ (note that $M$ is a non-Hermitian operator whose columns add up to zero). With the aid of this left state and starting from an initial probability distribution $|P(0)\rangle$, typical quantities of interest, such as the interface width $W^{2}$, are calculated as $\left\langle\widetilde{\psi}\left|\hat{\mathcal{W}} e^{-M t}\right| P(0)\right\rangle[17]$. Here, the "width operator" $\hat{\mathcal{W}}$ is obtained by promoting the slopes of Eq. (5) to $\sigma^{z}$ matrices.

Despite the apparent simplicity of our Metropolis operator, the $A$ 's above cannot be associated to elementary excitations of any kind and exact analytic treatments may seem unlikely. Nevertheless, Eq. (12) will permit some numerical progress on finite size systems after considering a simple transformation to be discussed later on in Sec. II C. Before that and for the sake of completeness, we pause to digress briefly about symmetries and conservation laws of $M$.

\section{B. Excursus: Constants of Motion}

Here we follow Refs. $[18,19]$ in closely related processes. Recalling that under a rotation by an angle $\theta$ around the $z$ direction $\sigma^{ \pm}$transform as $e^{ \pm i \theta} \sigma^{ \pm}$, we can therefore choose angles $\theta_{\alpha}$ for all spins in each sublattice $\Lambda_{\alpha}$ such that Eq. (10) [and obviously Eq. (11)] is left invariant. Clearly, this is the case of

$$
\sum_{\alpha}(-1)^{\alpha} \theta_{\alpha}=0 .
$$

On the other hand, the infinitesimal generator of this transformation is $S=\Sigma_{\alpha}(-1)^{\alpha} \theta_{\alpha} S_{\alpha}^{z}$, with $S_{\alpha}^{z}=\Sigma_{j \in \Lambda_{\alpha}} \sigma_{j}^{z}$. Since $M$ $=e^{i S / 2} M e^{-i S / 2}$, then $[M, S]=0$, and therefore $S$ is preserved by $M$. But from the constraint (13) it follows that $S$ can be rewritten in terms of three independent angles, that is, $S$ $=\Sigma_{\alpha \neq \alpha^{\prime}} \theta_{\alpha}\left[(-1)^{\alpha} S_{\alpha}^{z}-(-1)^{\alpha^{\prime}} S_{\alpha^{\prime}}^{z}\right]$ from which one recovers the three conserved quantities identified before on more intuitive grounds.

These continuous symmetries entail a number of invariant subspaces growing at most as $L^{3}$, which, however, by no means exhaust all possibilities. For instance, it is straightforward to see that already the number of jammed configurations (i.e., states that cannot evolve further), grows exponentially with the system size [18]. This unusual proliferation of invariant states should be the consequence of a much higher symmetry of $M$. Although its explicit operational form might be difficult to figure out, we can nevertheless follow Ref. [19] and construct an exponential number of dynamically disjoint sectors, either jammed or unjammed, regardless of the value of $\kappa$. To this end, one defines a reduction rule by looking at the occurrence of groups of active quartets in a given configuration $|s\rangle$. Each occurrence, if any, is deleted so the length of the remaining object is reduced in four bits per deletion. This procedure is applied recursively until one is left with a string that cannot be further reduced, i.e., an irreducible string $I\{s\}$. In turn, the result is unique irrespective of the order of deletion. To mention only a few examples: $I\{|\uparrow \downarrow \uparrow \downarrow \uparrow \downarrow \uparrow \downarrow \downarrow \uparrow\rangle\}=\downarrow \uparrow$ (either in one or two steps); the flat interface or antiferro state yields a null string, whereas any jammed configuration is already an irreducible string of length $L$. The key issue to bear in mind is that two states $|s\rangle$ and $\left|s^{\prime}\right\rangle$ belong to the same $M$ subspace $\Leftrightarrow I\{s\}=I\left\{s^{\prime}\right\}$ [19]. So, this nonlocal construct picks out both the length $\mathcal{L}=L$ $-4 k$ and the sequence of the irreducible string's elements (their combinations growing exponentially in $\mathcal{L}$ ), and ultimately defines the constant of motion under which the $M$ dynamics take place.

That being said, from now on we shall content ourselves with studying just the null string subspace selected by initially flat conditions, for the most part quite natural in the context of growing interfaces. Note also that the equilibrium properties of the $1 D$ Hamiltonian (4) are neither analytically simple to evaluate [e.g., Eq. (5), not even for $\kappa=0$ ], as the ensemble of averaged states must be consistent not only with $S^{z}=0$ (PBC) but with a vanishing irreducible string as well (totally unjammed conditions), which rules out an exponential number of states.

\section{Symmetric representation}

Returning to the discussion of Sec. II A, we may make some progress on the numerical analysis of $M$ by performing a similarity transformation so as to map this operator into an Hermitian matrix. This is feasible because detailed balance in rates (6) ensures the existence of a representation in which the evolution operator is self-adjoint [17]. For this purpose, it suffices to consider a diagonal transformation like the one discussed in Sec. II B but using pure imaginary angles instead. Specifically, we rotate each $j$ th spin around the $z$ direction by a site dependent angle (field operator)

$$
\varphi_{j}=\frac{i \kappa}{2}\left(\sigma_{j-1}^{z}+\sigma_{j+1}^{z}\right)
$$

by means of the nonunitary similarity transformation $U$ $=e^{-i \Sigma_{j} \varphi_{j} \sigma_{j}^{z} / 2}$. Under this rotation $\sigma_{j}^{ \pm} \rightarrow e^{ \pm(\kappa / 2)\left(\sigma_{j-1}^{z}+\sigma_{j+1}^{z}\right)} \sigma_{j}^{ \pm}$, so it is simple to check that the double hopping operators of Eq. (10) transform as

$$
\begin{aligned}
U A_{j}^{\dagger} U^{-1} & =e^{(\kappa / 2)\left(\sigma_{j-1}^{z}-\sigma_{j+4}^{z}\right)} A_{j}^{\dagger}, \\
U A_{j} U^{-1} & =e^{-(\kappa / 2)\left(\sigma_{j-1}^{z}-\sigma_{j+4}^{z}\right)} A_{j} .
\end{aligned}
$$

This introduces new diagonal operators $\hat{d}_{j}$ in the $\sigma^{z}$ representation, that like the $g_{j}^{ \pm}$operators commute with $A_{j}^{\dagger}, A_{j}$, and in terms of which the off diagonal part of $M$ becomes symmetric. More specifically, this symmetrization is produced by defining

$$
\hat{d}_{j}=e^{(\kappa / 2)\left(\sigma_{j-1}^{z}-\sigma_{j+4}^{z}\right)} g_{j}^{+}=e^{-(\kappa / 2)\left(\sigma_{j-1}^{z}-\sigma_{j+4}^{z}\right)} g_{j}^{-},
$$

their diagonal elements being $e^{-(\kappa / 2)\left|s_{j-1}-s_{j+4}\right|}$. As a result, Eq. (9) is transformed into $-\sum_{j} \hat{d}_{j}\left(A_{j}^{\dagger}+A_{j}\right)$ while Eq. (11) is left unchanged, so the rotated Metropolis operator $H=U M U^{-1}$ can be finally cast in the symmetric form

$$
H=\sum_{j}\left(A_{j}^{\dagger}+A_{j}\right)\left(g_{j}^{-} A_{j}^{\dagger}+g_{j}^{+} A_{j}-\hat{d}_{j}\right) .
$$

Consequently, the time dependent probability distribution turns out to be a superposition of orthogonal eigenlevels 
$\left|\psi_{\lambda \neq 0}\right\rangle$ with real eigenvalues $\lambda>0$ of $H$, each having typical lifetimes $1 / \lambda$. In particular, the ground state $\left|\psi_{0}\right\rangle$ has eigenvalue $\lambda=0$, and is just the transformed Boltzmann distribution $\left|\Psi_{0}\right\rangle$ referred to above, i.e., $\left|\psi_{0}\right\rangle=U\left|\Psi_{0}\right\rangle$ $\propto \sum_{s} e^{-(\kappa / 4) \sigma_{\{s\}}|S\rangle}$. Since left and right levels now coincide, it is thereby a simple matter to check that in the symmetric representation the dynamic of any diagonal observable, say the interface width $\hat{\mathcal{W}}_{L}=\frac{2}{L^{2}} \sum_{i<j} i(L-j) \sigma_{i}^{z} \sigma_{j}^{z}$ (in turn invariant under $U$ ), can be written as

$$
W^{2}(L, t)=W_{e q}^{2}+\sum_{\lambda_{L}>0} e^{-\lambda_{L} t}\left\langle\psi_{0}\left|\hat{\mathcal{W}}_{L}\right| \psi_{\lambda_{L}}\right\rangle\left\langle\psi_{0} \mid P^{\prime}(0)\right\rangle,
$$

where $W_{e q}^{2}$ is the saturation width reached at equilibrium, whereas $\left|P^{\prime}(0)\right\rangle=U|P(0)\rangle$ denotes the transformed initial distribution. From here we see that if the spectrum gap vanishes as $1 / L^{z}$, then a finite size scaling analysis of the first excited levels $\lambda_{L}$ will provide the dynamic $z$-exponent ruling over the late roughening stages referred to in Sec. I.

It is worth pointing out that the discussion presented so far can be readily extended to include monomers $(m=1)$, trimers $(m=3)$, etc., so long as the operators involved in Eq. (17) are reinterpreted as

$$
\begin{gathered}
A_{j}^{\dagger}=\prod_{i=1}^{m} \sigma_{j+2 i-2}^{+} \sigma_{j+2 i-1}^{-}, \\
g_{j}^{ \pm}=\min \left\{e^{ \pm \kappa\left(s_{\left.j+2 m^{-}-s_{j-1}\right)}\right.}, 1\right\}, \\
\hat{d}_{j}=e^{-(\kappa / 2)\left|s_{j-1}-s_{j+2 m}\right|},
\end{gathered}
$$

In particular, for monomers with no surface tension $(\kappa=0)$ the evolution operator reduces to the fully isotropic Heisenberg ferromagnet, thus recovering the usual EW dynamic exponent $z=2$. Among other numerical aspects, in what follows we shall focus on the evaluation of this quantity at $\kappa$ $\geqslant 0$ for both $m=1$ and 2 .

\section{NUMERICAL RESULTS}

The above ideas provide an alternative manner to evaluate dynamic exponents, independently of those obtained by the application of the dynamic scaling hypothesis (1). Thus, we first explore the consequences arising from the exact diagonalization of Eq. (17) in small systems, and then go on to corroborate them over larger length scales [via Eq. (1)] using standard Monte Carlo simulations. In addition, these latter will complement the evaluation of $z$ in low temperature regimes where, as we shall see, resorting to small lattice sizes might become inadequate.

\section{A. Scaling the gap}

To analyze our stochastic matrix we first obtained its nullstring basis using dimers on rings of sizes $L=4 k$. This was easily implemented by applying $H$ (or alternatively, $M$ ) to either of the two antiferro states and keeping proper track of the new generated configurations. By iterating this procedure with those new states for which $H$ was not previously ap-

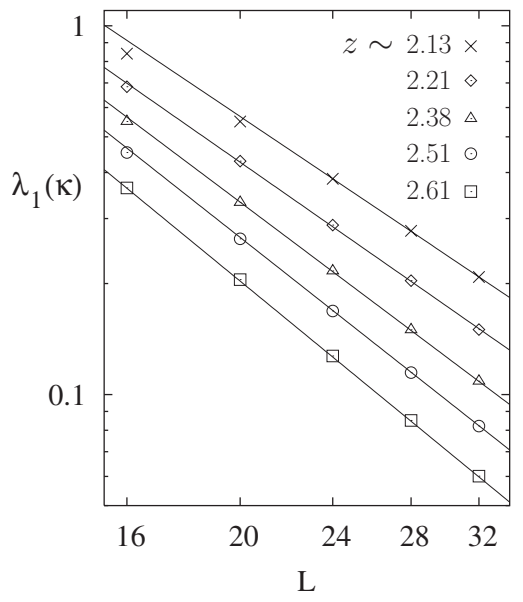

FIG. 2. Finite size behavior of the first excited level $\lambda_{1}$ of the Metropolis operator (17). The inverse temperature $\kappa$ decreases from top to bottom, each symbol standing, respectively, for $\kappa$ $=1, \frac{2}{3}, \frac{2}{5}, \frac{1}{5}, 0$. The dynamic exponents $z$ are read off from the slopes of the fitting lines.

plied, the whole null-string subspace was finally expanded. The total number of states so found is of course independent of $\kappa$, and as expected (see Sec. II B), grows slower than the monomer space dimensionality $\left(\begin{array}{c}L \\ L / 2\end{array}\right) \propto 2^{L}$. More specifically, this dimension seems to increase as $\approx 1.6(7)^{L}$, at least for the sizes at hand, which in turn allowed us to explore rings of up to 32 sites [20].

Once having identified the null-string configurations, we proceeded to evaluate exactly the low lying levels of $H$ (in principle, just the first excited will do), via a recursion type Lanczos algorithm [16]. Starting that recursion from a random linear combination of null strings but chosen orthogonal to the Boltzmann type distribution $\left|\psi_{0}\right\rangle$ referred to above, we then obtained the finite size behavior of the dimer gap, i.e., of $\lambda_{1}$. This is shown in Fig. 2 for several temperatures within the range $0 \leqslant \kappa \leqslant 1$ indicating a gap decrease $\propto L^{-z}$, however, notice that the data do not fall into parallel straight lines. In an attempt to standardize this situation we used scalings of the form $\lambda_{1}(L)=A_{\kappa} L^{-z_{1}}+B_{\kappa} L^{-z_{2}}$, but a large amount of uncertainty in both $z_{1}$ and $z_{2}$ raised doubts about the adequacy of such a procedure. Also, logarithmic corrections were attempted but no evidence supporting these latter were found. Thus, in principle we are led to suggest a plain power law decay although with a nonuniversal temperature dependent dynamic exponent $z=z(\kappa)$. In particular, $z(0) \sim 2.6(1)$ is in fair agreement with the value obtained in Ref. [5] by standard simulations of dimer interfaces without surface tension.

A slightly improved estimation of $z$ can be made by defining an effective dynamic exponent

$$
z_{L}=\frac{\ln \left[\lambda_{1}(L-4) / \lambda_{1}(L)\right]}{\ln [L /(L-4)]},
$$

and then extrapolating $z_{L}$ to $L \rightarrow \infty$ for a given $\kappa$. The results of this are exhibited in Fig. 3, which for comparison also displays the corresponding monomer dynamic exponents. These latter were derived using Eq. (19) for $m=1$ along with a similar numerical analysis but employing $L=2 k(\leqslant 24)$ in- 


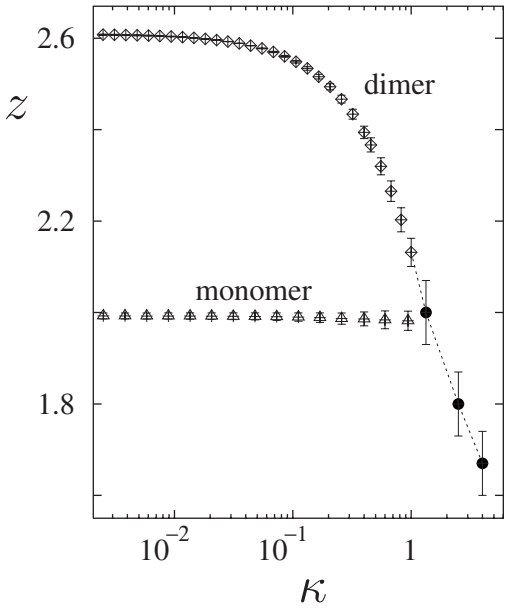

FIG. 3. Nonuniversal exponents of the dimer dynamics. Rhomboids indicate the results obtained from finite size scaling extrapolations of Metropolis gaps (see Fig. 2). For comparison, the triangles exhibit these exponents in the monomer case (close to $z$ $\approx 2$ ). Filled circles (joined by dotted lines) denote the $z$ values arising from the dynamic scaling hypothesis (1) applied to much larger systems at lower temperatures.

stead. Clearly, an EW behavior characterized by the Heisenberg exponent $z=2$ mentioned a little earlier can be discerned in monomer interfaces, as opposed to dimer exponents, which evidently are nonuniversal, at least if we are to judge by their $\sim 20 \%$ variation between $\kappa \approx 0$ and 1 . Although it is true that size effects increase monotonically our error margins with $\kappa$, they are nevertheless fairly bounded within the range inspected (see also Fig. 2).

A measure of these size effects is provided by the equilibrium correlation length of the associated Ising antiferromagnet appearing in Eq. (4). If this length becomes comparable to our available sizes, particularly at low temperatures, then the asymptotic dynamics will be distorted on approaching equilibrium as the average antiferromagnetic domain sizes, representing active regions in the interface, will be cut off by $L$. This situation is illustrated in Fig. 4 where we show the pair correlations $C(r)=\frac{1}{L} \Sigma_{j}\left\langle\sigma_{j}^{z} \sigma_{j+r}^{z}\right\rangle$ evaluated in the

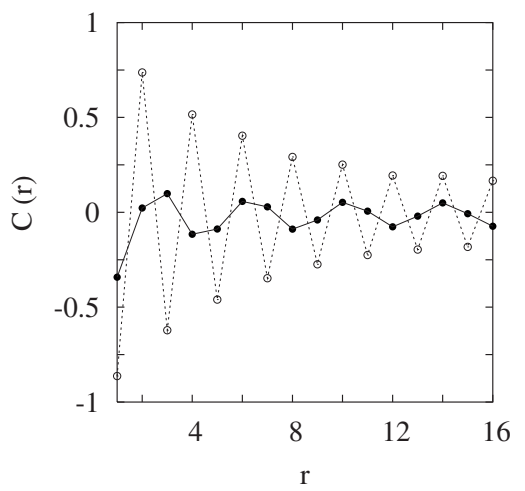

FIG. 4. Pair correlations in the ground state of operator (17) for 32 spins. Filled circles $(\kappa=0)$ are representative of high temperature regimes (which are quantitatively similar up to $\kappa \approx 1$ ). Above $\kappa$ $=2$ (open circles), the underlying correlation lengths become rapidly comparable to the lattice size.

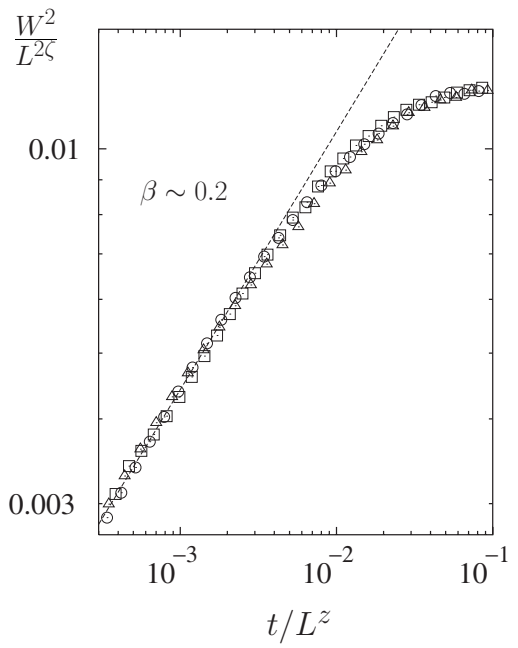

FIG. 5. Dynamic scaling of the dimer interface width [Eq. (1)] taking $\kappa=4$. Sizes $L=2^{12}, 2^{11}$, and $2^{10}$ are denoted, respectively, by triangles, circles, and squares. The data collapse was attained upon setting $\zeta \approx 0.3(4)$ and $z \approx 1.6(7)$. The dashed line is fitted with slope $2 \beta=2 \zeta / z$.

ground state or equilibrium distribution of $H$ [as stressed above, notice that analytic treatments are difficult even in this simpler case because of the null string constraint imposed on Eq. (4)]. In between $0 \leqslant \kappa \leqslant 1$, traces of antiferromagnetic short range order are nearly smeared out and correlation lengths become small. However, above $\kappa \approx 2$ they rapidly grow up and eventually get comparable to our maximum sizes, so precluding further analyses of the gap (which in fact comes out to be almost size independent). Thus, to complement the results obtained so far and check whether nonuniversal exponents actually extend down to low temperature regimes, we finally turn to the dynamic scaling hypothesis and simulations using larger substrates.

\section{B. Simulations}

Following the Metropolis rules referred to in Sec. II, we evolved initially flat interfaces with $L=2^{10}, 2^{11}$, and $2^{12}$ heights until reaching their stationary states. After a sequence of $L$ update attempts at random locations, the time scale was increased in one unit, i.e., $t \rightarrow t+1$, irrespective of these attempts being successful or not. Measurements of $W(t)$ were carried out for $\kappa=1.3,2.5,4$ and were averaged typically over $10^{4}$ independent histories. In Fig. 5 we display one of the characteristic scaling curves obtained using Eq. (1) for $\kappa=4$. There, the data collapse was attained by setting roughening exponents $\zeta \approx 0.3(4)$, which are practically common to all temperatures studied (see also Fig. 7 below). By contrast, this is not the case of the $z$ exponents which, in line with the results of Sec. III A, are severely altered by $\kappa$. Although their precise values are blurred by our not too sensitive collapse conditions, nevertheless they do follow the nonuniversal trend already found with our gap analysis, as can be seen in Fig. 3.

To corroborate further the validity of this claim, we also conducted simulations in much bigger scales measuring di- 


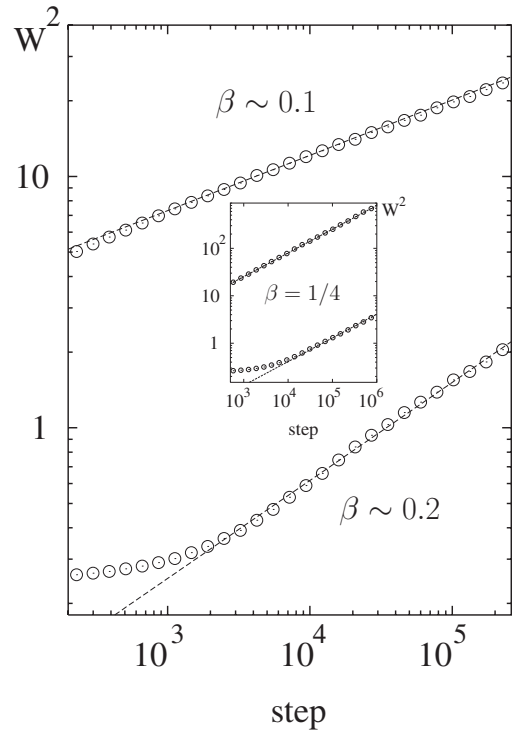

FIG. 6. Growth of interface width for $10^{6}$ sites at $\kappa=0$ (upper curve), and $\kappa=6$. Dashed lines are fitted with slopes $2 \beta$. The inset displays the corresponding situations for monomer growing interfaces.

rectly the growth exponent $\beta \equiv \zeta / z$. The reader's attention is now directed to Fig. 6 where the width evolution is contrasted at high and low temperature regimes in substrates of $10^{6}$ sites. As expected, nonuniversal aspects show up: after averaging over $\sim 40$ histories, clearly two rather different $\beta$ exponents emerge and hold for at least two decades. On the other hand, using the universal roughening exponent $\zeta$ $\approx 1 / 3$ already identified (see further estimations below), we thus obtain values of $z$ consistent with those previously encountered in smaller systems. For comparison, the inset of Fig. 6 also shows the typical EW $\beta$ values of the corresponding monomer cases which, alike their dynamical exponents in Fig. 3, remain robust under wide temperature intervals.

In respect of the roughening exponents, we finally considered the saturation or equilibrium widths $W_{e q}$ of a variety of substrate sizes subject to $\kappa=0,1,2$, and 4 . The employed relaxation times $\propto L^{z(\kappa)}$, range from $2 \times 10^{6}-10^{5}$ Monte Carlo steps for the largest cases and, as expected, decrease monotonically with $\kappa$. Our results are displayed in Fig. 7, clearly suggesting a common value of $\zeta$. Due to the pair correlations involved in $W_{e q}$ [see Eq. (5)], here size effects are also more noticeable at low temperatures. Nonetheless, a simple numerical fit of both amplitudes and slopes indicates that most of our data $(L \gtrsim 200)$ can be accounted for by the parametrization

$$
W_{e q} \approx 0.4(2) e^{-\kappa \zeta} L^{\zeta},
$$

with $\zeta \approx 0.3(2)$. This means that in equilibrium the interface becomes actually rough so long as $\ln L \gg \kappa$ is held in the thermodynamic limit.

\section{CONCLUDING DISCUSSION}

To summarize, we have studied numerically the dynamics of dimer growing interfaces at finite temperatures using two

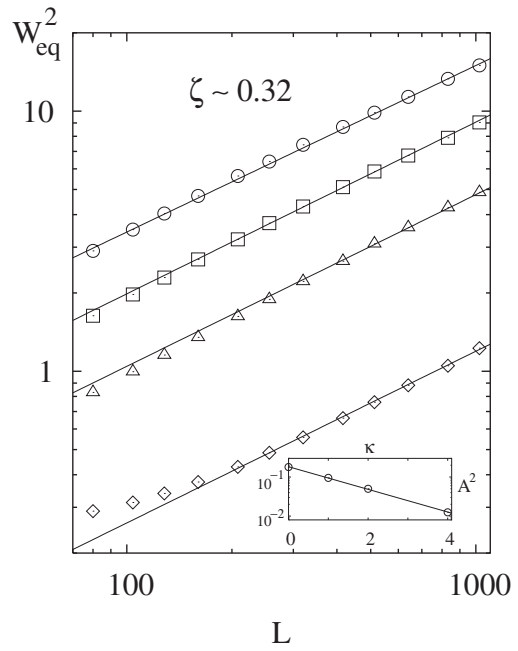

FIG. 7. Finite size behavior of the saturation width. The symbols stand in turn for $\kappa=0$ (circles), $\kappa=1$ (squares), $\kappa=2$ (triangles), and $\kappa=4$ (rhomboids). Solid lines display a common slope $2 \zeta$. As is shown in the inset, the amplitudes of these latter exhibit an exponential decay with slope $-2 \zeta$ [see Eq. (21)].

independent procedures. The first one analyzes the spectrum gap of the evolution operator [Eq. (17)] by exact diagonalization of small systems, thus picking out dynamic exponents in a direct manner. Clear finite size trends were obtained in the range $0 \leqslant \kappa \leqslant 1$ (Fig. 2), and fairly bounded extrapolations were derived for $z$ (Fig. 3). Although the nonlocal symmetries (i.e., irreducible strings of Sec. II B) of our stochastic operators are unaffected by surface tensions, surprisingly, the $z$ exponents are nonuniversal, being dependent on $\kappa$. Yet, a theoretical interpretation of such puzzling behavior remains quite open. This is in marked contrast with the dynamics of monomers interfaces, as their tensions do not take over neither the EW nor the KPZ universality classes, at least in $1+1$ dimensions [11].

Secondly, using the standard scaling hypothesis [Eq. (1), Fig. 5] we checked out these findings under lower temperature regimes where correlation lengths become larger than our maximum diagonalizable sizes (Fig. 4). Despite the limited precision of this method for $\kappa>0$, our results confirmed the nonuniversal tendency observed in Sec. III A. In turn, measurements of growth exponents in much larger substrates (Fig. 6) further validated the monotonic decrease of $z(\kappa)$.

As for the roughening exponents (Fig. 7), in all studied cases with $\kappa>0$ the global constraint referred to in Sec. I and further examined in terms of irreducible strings $[18,19]$, led to anomalous motions of a rather unconventional type $(\zeta \approx 1 / 3)$, as compared to Levi flights and other restricted random paths [21]. More specifically, they are consistent with those of even visiting random walks [3] and not comprehensible in terms of EW or diffusive interfaces (e.g., monomers), which are definitely rougher. Under surface tension the range of correlations so introduced in the associated walk is finite, and therefore the scaling of its width must remain unchanged (consult Ref. [21]), though in line with Eq. (21), its proportionality constant might depend on the precise form of these correlations. To endow further this robustness of $\zeta$, it would be interesting to elucidate whether the 
analogy of noninteracting electrons moving in a random medium studied in Ref. [3] could be extended to the finite temperature interfaces (walks) investigated here.

Other pending issues of interest concern starting the growth process from more general initial conditions (i.e., not in the null string sector), capable of modifying asymptotic regimes [5], as well as considering biased dynamics (without detailed balance) such as those analyzed in Refs. [11,12]. In principle, the first situation could also be studied with the methodology of Sec. II A; however, for the second one the similarity transformation of Sec. II C is no longer useful and the unsymmetrization of the Lanczos recursion would be inevitable [16]. Finally, 2+1 dimensional generalizations of this study could shed light on the combined role that dimer dynamics and substrate geometry might have in catalytic processes. Whether or not nonuniversal aspects would also emerge there under surface tension, deserves further investigations.

\section{ACKNOWLEDGMENTS}

The author is grateful to R. B. Stinchcombe for helpful observations and correspondence. Support of CONICET, Argentina, under Grants No. PIP 5037 and No. PICT ANCYPT 20350, is acknowledged.
[1] For comprehensive reviews and a literature list consult P. Meakin, Fractals, Scaling, and Growth Far from Equilibrium (Cambridge University Press, Cambridge, England, 1998); J. Krug, Adv. Phys. 46, 139 (1997); T. Halpin-Healy and Y.-C. Zhang, Phys. Rep. 254, 215 (1995).

[2] F. Family and T. Vicsek, J. Phys. A 18, L75 (1985).

[3] J. D. Noh, H. Park, D. Kim, and M. den Nijs, Phys. Rev. E 64, 046131 (2001); J. D. Noh, H. Park, and M. den Nijs, Phys. Rev. Lett. 84, 3891 (2000).

[4] H. Hinrichsen and G. Ódor, Phys. Rev. Lett. 82, 1205 (1999); Phys. Rev. E 60, 3842 (1999).

[5] M. D. Grynberg, J. Stat. Phys. 103, 395 (2001).

[6] D.-S. Lee and M. den Nijs, Phys. Rev. E 65, 026104 (2002).

[7] S. F. Edwards and D. R. Wilkinson, Proc. R. Soc. London, Ser. A 381, 17 (1982).

[8] M. Kardar, G. Parisi, and Y.-C. Zhang, Phys. Rev. Lett. 56, 889 (1986).

[9] Y. Kim and S. Y. Yoon, Phys. Rev. E 69, 027101 (2004); 72, 012601 (2005).

[10] Y. Kim, T. S. Kim, and H. Park, Phys. Rev. E 66, 046123 (2002); Y. Kim, H. B. Heo, and S. Y. Yoon, ibid. 68, 026108 (2003)

[11] J. G. Amar and F. Family, Phys. Rev. Lett. 64, 543 (1990); D. A. Huse, J. G. Amar, and F. Family, Phys. Rev. A 41, 7075 (1990).

[12] J. Krug and H. Spohn, Phys. Rev. Lett. 64, 2332 (1990).

[13] P. M. Chaikin and T. C. Lubensky, Principles of Condensed
Matter Physics (Cambridge University Press, Cambridge, England, 1995), see chapter 10; Consult also M. den Nijs, Phys. Rev. Lett. 64, 435 (1990); M. den Nijs and K. Rommelse, Phys. Rev. B 40, 4709 (1989).

[14] P. Meakin, P. Ramanlal, L. M. Sander, and R. C. Ball, Phys. Rev. A 34, 5091 (1986); M. Plischke, Z. Rácz, and D. Liu, Phys. Rev. B 35, 3485 (1987).

[15] R. B. Stinchcombe, Adv. Phys. 50, 431 (2001); G. M. Schütz, in Phase Transitions and Critical Phenomena, C. Domb and J. L. Lebowitz eds. (Academic, London, 2001).

[16] See, for example, G. H. Golub and C. F. van Loan, Matrix Computations, 3rd. ed. (Johns Hopkins University Press, Baltimore, 1996).

[17] N. G. van Kampen, Stochastic Processes in Physics and Chemistry, 2nd ed. (North Holland, Amsterdam, 1992).

[18] M. Barma, M. D. Grynberg, and R. B. Stinchcombe, Phys. Rev. Lett. 70, 1033 (1993); R. B. Stinchcombe, M. D. Grynberg, and M. Barma, Phys. Rev. E 47, 4018 (1993).

[19] D. Dhar and M. Barma, Pramana, J. Phys. 41, L193 (1993); M. Barma and D. Dhar, Phys. Rev. Lett. 73, 2135 (1994).

[20] Had the restricted SOS constraint $\Delta h=0, \pm 1$ been chosen, it would immaterially complicate the numerical analysis demanding instead spin-1 operators.

[21] M. N. Barber and B. W. Ninham, Random and Restricted Walks, Theory and Applications (Gordon and Breach, New York, 1970); see chapters 6 and 7. 